Math. Model. Nat. Phenom.

Vol. 3, No. 7, 2008, pp. 90-114

\title{
Reaction-difusion model of early carcinogenesis: The effects of influx of mutated cells
}

\author{
Anna Marciniak-Czochra ${ }^{a 1}$ and Marek Kimmel ${ }^{b, c}$ \\ ${ }^{a}$ Institute of Applied Mathematics, University of Heidelberg, 69120 Heidelberg, Germany \\ ${ }^{b}$ Department of Statistics, Rice University, Houston, TX 77005, USA \\ ${ }^{c}$ Systems Engineering Group Silesian University of Technology, 44-100 Gliwice, Poland
}

\begin{abstract}
In this paper we explore a new model of field carcinogenesis, inspired by lung cancer precursor lesions, which includes dynamics of a spatially distributed population of pre-cancerous cells $c(t, x)$, constantly supplied by an influx $\mu$ of mutated normal cells. Cell proliferation is controlled by growth factor molecules bound to cells, $b(t, x)$. Free growth factor molecules $g(t, x)$ are produced by precancerous cells and may diffuse before they become bound to other cells. The purpose of modelling is to investigate the existence of solutions, which correspond to formation of multiple spatially isolated lesions of pre-cancerous cells or, mathematically, to stable spike solutions. These multiple lesions are consistent with the field theory of carcinogenesis. In a previous model published by these authors, the influx of mutated cells was equal to zero, $\mu=0$, which corresponded to a single pre-malignant colony of cells. In that model, stable patterns appeared only if some of the growth factor was supplied from outside, arguably, a biologically tenuous hypothesis. In the present model, when $\mu>0$, that hypothesis is no more required, which makes this model more realistic. We present a range of results, both mathematical and computational, which taken together allow understanding the dynamics of this model. The equilibrium solutions in the current model result from the balance between new premalignant colonies being initiated and the old ones dying out.
\end{abstract}

Key words: cancer modelling, cooperation, reaction-diffusion equations, pattern formation, spike solutions.

AMS subject classification: 35K57, 35B35, 35B40, 92C37, 92C50.

\footnotetext{
${ }^{1}$ Corresponding author. Email: anna.marciniak@iwr.uni-heidelberg.de
} 


\section{Introduction}

\subsection{Field theory and other theories of carcinogenesis}

Field carcinogenesis states that as a result of exposure to carcinogens and/or of inherited genetic variants (mutations), a substantial portion of an organ (called the field) can be enriched in genetic variants of cells, which then may or may not acquire further genomic modifications. These modifications will result in increased proliferation and invasion of the surrounding tissues. The rearrangements involved may have different nature: They may range from accumulation of point mutations or microsatellite variability, to gene amplification, and to changes in DNA content, number and organisation of chromosomes (aneuploidy and translocation). Therefore, although they may form a linear irreversible progression, it is more likely that they form a more complex network. Because of the spatial dimensions of the field, emerging groups of transformed cells (precancerous and early cancerous tumours; $[10,11])$ will represent different levels of transformation, and may exhibit both progression and reversals. They will be frequently multifocal. This viewpoint is in opposition to the clonal theory of carcinogenesis, which assumes linear irreversible progression and generally unifocal lesions.

Let us consider a sample scenario of events, which might be partly corroborated by published evidence. Suppose that cells of some type in a region of an organ have (as a result of inheritance or exposure) an ineffective variant of a gene (such as the p53 gene) providing a cell cycle checkpoint. The normal variant of this gene does not allow cells to enter division if unrepaired damage in DNA is detected. However the mutant gene will allow this, and the cells will go through a series of divisions, which may be not exactly symmetric, so that part of the progeny will be deprived of some other genes and part will have these genes amplified (increased number of gene copies) [1,23]. Gradually, this will relax the controls on correct segregation of chromosomes and lead to aneuploidy (irregular amount of DNA in the cells [21]). Because of increase in the number of divisions, the telomeres in these partly transformed cells will shorten, which will further impair correct segregation [7].

It has to be noted that it is unlikely to expect that in each cell lineage, these sequence of events will be identical. Therefore, the field will frequently give rise to differently transformed (genetically distinct) groups of cells (non-clonality [18]).

Another important aspect of early carcinogenesis is signalling and production of growth factors. It has been recently proposed [2] that early carcinogenesis may be a result of cooperative interactions between partly progressed cell populations, which produce growth factors in a complementary way, for example cells of type 1 produce growth factor needed by cells of type 2 and vice versa. Although this theory has experimental foundations, it is missing the dynamic transformation element prominent in field carcinogenesis.

One of the important elements to be taken into account, is the spatial diffusion of growth factors and other signalling molecules. As it is known, diffusion in connection with nonlinear kinetics and growth regulation may lead to instability and pattern formation. This is an important prediction, since it suggests that in field carcinogenesis, diffusion of free 
growth factor molecules leads to diversified multifocal lesions arising on the background of primed tissue, the exact pattern of which depends on chance and small fluctuations of initial conditions.

\subsection{The $\mathrm{AAH} \rightarrow \mathrm{BAC} \rightarrow$ adenocarcinoma sequence in lung cancer}

A specific biological example motivating our model is the atypical adenomatous hyperplasia (AAH), which is considered a candidate precursor lesion for adenocarcinoma of the lung [8]. As illustrated in Figure 1A in [8], also reproduced in [13], the AAH lesions are placed along the walls of smallest branchings of the bronchial tree (the alveoli). Citing from [8]: From a morphological perspective there seems little doubt that AAH might progress and develop, through a stage of bronchoalveolar adenocarcinoma (BAC), into invasive adenocarcinoma. AAH lesions are usually invisible to the naked eye, but the larger ones or those with more fibrosis in their walls may be visible on the cut surface of well inflated and formalin fixed lung. In the process of progression, the tubular (or approximately linear) AAH structures thicken in places and invade the surrounding tissue.

\subsection{Modelling early carcinogenesis}

In a series of recent papers $[12,13,14]$ we analysed models of spatially-distributed growth of clonal populations of pre-cancerous cells, which remained under control of endogenous or exogenous growth factors diffusing in the extracellular medium and binding to cell surface. In these models, which had the mathematical form of mixed reaction-diffusion and ordinary differential equations, we found conditions for emergence of growth patterns concentrated around discrete points along the spatial coordinate, which took the mathematical form of spike-type spatially inhomogeneous steady states. This multifocality is as expected from the field theory of carcinogenesis.

By a combination of analytic and computational methods, we investigated the dynamics of these emerging patterns. The general conclusion was that although the clonal populations exhibited multifocal growth patterns, these in turn might either lead to decay of the cell population or to establishment of stable cell foci. The dynamics depended on supply of free growth factor particles. If these were supplied from outside, stability emerged, whereas if they were produced by premalignant cells themselves, only a transient growth followed by decay was observed. This was only partly remedied by combining the model with the cooperative mechanisms postulated by Axelrod and others [2, 12].

In the current paper, we consider the more biologically realistic situation in which a constant influx of pre-cancerous cells is provided from mutation of normal cells. We will demonstrate, among other, that this hypothesis leads to computationally stable multifocalities, under conditions, which seem biologically justifiable. In particular, the assumption of external supply of growth factors is not required. 


\section{The model with mutated cell influx}

\subsection{Derivation of the model}

The model of a precancerous cell population adopts some elements of the model of a homogeneous population of cells of one type we previously proposed $[12,13,14]$. The present model is based on the following hypotheses:

- Precancerous cells $c$, existing in a spatial domain, proliferate at a rate $a(b, c)$, which is reduced by cell crowding but enhanced in a paracrine manner by a hypothetical biomolecular growth factor $b$ bound to cells.

- Free growth factor $g$ is secreted by the cells at the rate $\kappa(c)$, then it diffuses among cells with diffusion constant $D$, and binds to cell membrane receptors at a rate $\alpha(c)$, becoming the bound factor $b$. It then dissociates at a rate $d$, returning to the $b$-pool.

- Free and bound growth factor particles decay at rates $d_{g}$ and $d_{b}$, respectively.

Discussion of possible geometries for the spatial variable $x$ can be found in previous papers $[13,14]$. One natural geometry is that of a line of cells, occupying the interval $x \in[0, L]$, There are three substances distributed over the line's length, cells, free and bound growth factor molecules, with densities $c(x, t), g(x, t)$ and $b(x, t)$, respectively. The resulting equations are identical as in our recent paper [12] except that now the pre-cancerous cells are supplied at a constant rate $\mu$ by mutation of normal cells

$$
\begin{aligned}
& \frac{\partial c}{\partial t}=\left(a(b, c)-d_{c}\right) c+\mu \\
& \frac{\partial b}{\partial t}=\alpha(c) g-d_{b} b-d b \\
& \frac{\partial g}{\partial t}=\frac{1}{\gamma} \Delta_{x} g-\alpha(c) g-d_{g} g+\kappa(c)+d b
\end{aligned}
$$

with homogeneous Neumann (zero flux) boundary conditions for $g$,

$$
\partial_{x} g(0, t)=\partial_{x} g(1, t)=0 .
$$

The kinetics were derived from the stochastic model describing the transitions between different states of the growth factor molecules [13, 14]. Diffusion equation is a macroscopic approximation of the microscopic process of growth factor binding to membrane receptors, under homogeneity hypotheses; it has been derived in [16]. Coefficient $1 / \gamma$ is a composite parameter including the diffusion constant and the domain scalling parameter, $\gamma=L^{2} / D$. Proliferation rate has the Hill function form

$$
a(c, b)=\frac{a_{1}(b / c)^{m}}{1+(b / c)^{m}},
$$


where $a_{1}=(2 p-1) a_{0}$ and $p$ is the eficiency of divisions. We will consider the special case $m=1$. Growth factor binding rate has the form

$$
\alpha(c)=\alpha c^{2}
$$

which follows from conditions for diffusion-driven instability further on. Production of free growth factor by cells has the Michaelis-Menten form

$$
\kappa(c)=\frac{\kappa c}{1+c} .
$$

This form of $\kappa$ reflects the assumption that there is no external supply of growth factor particles.

Remark 2.1. In the context of the specific form of nonlinearities used in the present model, the binding rate is assumed proportional to $c^{1+\varepsilon}$ (with $c^{2}$ being a computationally convenient special case). This assumption, which amounts to cooperativity in receptor binding, is necessary for diffusion-driven instability to emerge. It is not needed if other forms of nonlinearities in $a(c, g)$ are used (see [13]).

\subsection{Existence and boundedness of solutions}

The regularity of the model solutions depends on the regularity of the initial conditions. For the initial conditions in the spaces of continuously differentiable and $\alpha$-Hlder functions $c_{0}, b_{0} \in C^{\alpha}(\bar{\Omega})$ and $g_{0} \in C^{2+\alpha}(\bar{\Omega})$ there exists a local solution $(c, b, g) \in C^{\alpha, 1+\alpha / 2}(\bar{\Omega} \times[0, T]) \times$ $C^{\alpha, 1+\alpha / 2}(\bar{\Omega} \times[0, T]) \times C^{2+\alpha, 1+\alpha / 2}(\bar{\Omega} \times[0, T])$ for all $T \in\left(0, T_{\max }\right)$ of the initial value problem (2.1)-(2.3), see Ref. [20] for the proof.

Using the framework of invariant rectangles (see [22] and [3] for $\Omega \subset \mathbb{R}^{n}$ ) we can show that solutions of system (2.1)-(2.3) remain uniformly bounded and nonnegative for nonnegative initial conditions. The existence of global solutions results from a standard argument based on the theory of bounded invariant rectangles and a priori estimates [22].

\section{Qualitative behaviour of the model with cell influx}

We will explore a mechanism of pattern formation arising in the processes described by the model (2.1)-(2.3). The mechanism responsible for destabilisation of the stationary spatially homogeneous state and growth of spatially heterogeneous patterns is diffusion-driven instability (Turing-type instability). It occurs when there exists a spatially homogeneous solution, which is locally asymptotically stable in the space of constant functions, i.e., for the kinetics system, but it is unstable under spatially inhomogeneous perturbations. Existence and stability of spatially heterogeneous patterns arising in models exhibiting diffusion-driven instability, but consisting of a single reaction-diffusion equation coupled with a system of ODEs or even a single ODE is an interesting issue. Such models are very different from 
classical Turing-type models and the spatial structure of the pattern emerging from the destabilisation of the spatially homogeneous steady state cannot be concluded from a linear stability analysis $[15,13,19]$. They exhibit qualitatively new patterns of behaviour of solutions, including, in some cases, a strong dependence of the emerging pattern on initial conditions and quasi-stability followed by rapid growth of solutions $[15,14,13]$.

As mentioned in the Introduction, it follows from our previous analyses [12] that model (2.1)-(2.3) with $\mu=0$ can exhibit diffusion-driven instability, which in turn may lead either to decay of the cell population, or to the emergence of local growth foci, represented by spikelike solutions. The dynamics depends on the form of the function $\kappa$ describing the supply of free growth factor particles. Our analysis confirmed by numerical simulations showed that, with $\mu=0$, if $\kappa(0)=0$ then all the solutions tend to zero, while $\kappa(0)>0$ (and the parameters admitting diffusion-driven instability) leads to the formation of the stationary spike-like patterns.

Here, we aim to check how the assumption $\mu>0$ on the influx of new cells may change the dynamics of the model solutions. In particular, we show that the model with cell influx $\mu>0$ can exhibit the growth of spike-like patterns, even if $\kappa(0)=0$, i.e., without the assumption of external supply of growth.

\subsection{Diffusion-driven instabilities}

We start from the checking if the model (2.1)-(2.3) with $\mu>0$ may exhibit diffusion-driven instabilities (DDI). To this aim we consider bifurcation from the steady state by examining the response of the system to an initially small perturbation from the steady value. Stability of smooth stationary solutions of reaction-diffusion equations may be analysed by considering the eigenvalues of the linearised system (for a spectral criterion see e.g.,[4], and for the systems coupled with ODEs [17]). The dispersion relation, $\lambda=\lambda\left(\mu_{m}^{2} / \gamma\right)$, an algebraic equation describing the dependence of the growth rate $\lambda$ of the system with diffusion on the eigenvalues $\mu_{n}^{2}$ of the diffusion operator, indicates which eigenfunctions that is which spatial patterns, are linearly unstable and grow with time.

For a generic system of two ordinary differential equations coupled with a reactiondiffusion equation the following Proposition holds.

Proposition 3.1. (modified Lemma in [13]) Let us consider the system

$$
\left\{\begin{aligned}
\partial_{t} c & =F_{c}(c, b, g), \\
\partial_{t} b & =F_{b}(c, b, g) \\
\partial_{t} g & =\frac{1}{\gamma} \Delta_{x} g+F_{g}(c, b, g),
\end{aligned}\right.
$$

with $A=\left\{a_{i j}\right\}, i=1,2,3, j=1,2,3$ being the Jacobian matrix of the kinetics system of (3.7), computed at a positive spatially homogeneous steady state $(\bar{c}, \bar{b}, \bar{g})$, and such that

(i) the diagonal elements of $A$ are negative, i.e., $a_{i i}<0$ for $i=1,2,3$,

(ii) $a_{12} a_{21}>0$. 
There is a DDI for system (3.7) if and only if the following conditions are fulfiled,

$$
\begin{aligned}
-\operatorname{tr}(A) & >0 \\
-\operatorname{tr}(A) \sum_{i<j}\left|A_{i j}\right|+|A| & >0 \\
-|A| & >0 \\
\left|A_{12}\right| & <0
\end{aligned}
$$

where $A_{i j}$ is a submatrix of $A$ consisting of the $i$-th and $j$-th column and $i$-th and $j$-th row, and $|A|$ and $\left|A_{i j}\right|$ denote the determinants of matrices $A$ and $A_{i j}$, respectively.

Conditions (3.8)-(3.10) are necessary for the stability of the steady state in the absence of diffusion. Condition (3.11) is the sufficient and necessary condition for destabilisation of this steady state in the system without autocatalysis, i.e. under the assumption (i) that all $a_{i i}<0$ for $i=1,2,3$ and assumption (ii) that $a_{12} a_{21}>0$. Assumption (ii) is a straightforward consequence of the biological model (for the Proof see [13]).

Proposition 3.1 indicates that in the case of systems with one diffusion operator a spatially homogeneous steady state, if it exists and is stable in the absence of diffusion, is destablised if and only if the ODE subsystem is unstable. This result can be generalised to larger systems of ODEs coupled with one reaction-diffusion equation. For such system, the dispersion relation, $\lambda=\lambda\left(\mu_{m}^{2} / \gamma\right)$, which is an algebraic equation describing the dependence of the growth rate of the full system on the eigenvalues of the diffusion operator, reads

$$
\lambda\left(\frac{\mu_{m}^{2}}{\gamma}\right):|A|-\left|A_{12}\right| \frac{\mu_{m}^{2}}{\gamma}=0
$$

Condition $\lambda\left(\frac{\mu_{m}^{2}}{\gamma}\right)>0$ is verified if $\mu_{m}^{2} / \gamma$ is large enough. As a consequence, for every $\gamma$, there exist infinitely many different integer $\mu_{m}$ for which the above inequality is satisfied.

The consequence of the Proposition applied to model (2.1)-(2.3) is the following.

Theorem 3.2. The DDI for system (2.1)-(2.3) occurs if and only if there exists a nonnegative steady state $(\bar{c}, \bar{b}, \bar{g})$, which is stable to the spatially homogeneous perturbations, i.e., conditions (3.8)-(3.10) hold, and

$$
a_{1} \bar{c}^{2} \bar{b}\left[\alpha(\bar{c})-\left.\bar{c} \alpha^{\prime}(c)\right|_{\bar{c}}\right]+\mu(\bar{c}+\bar{b})^{2} \alpha(\bar{c})<0
$$

Proof. We calculate $\left|A_{12}\right|=a_{11} a_{22}-a_{12} a_{21}$ using the steady states conditions,

$$
\left\{\begin{aligned}
a_{1} \frac{\bar{b}}{\bar{c}+\bar{b}} & =d_{c}-\frac{\mu}{\bar{c}}, \\
\bar{g} & =\frac{\kappa(\bar{c})-d_{b} \bar{b}}{d_{g}}, \\
\alpha(\bar{c}) \bar{g} & =\left(d_{b}+d\right) \bar{b},
\end{aligned}\right.
$$

and conclude that $\left|A_{12}\right|<0$ if and only if (3.13) is satisfied. 
Remark 3.3. Condition (3.13) is satisfied only if

$$
\alpha(\bar{c})-\left.\bar{c} \alpha^{\prime}(c)\right|_{\bar{c}}<0,
$$

which is satisfied when function $\alpha$ assumes the form,

$$
\alpha(c)=\alpha_{1} c^{s+1}, \quad s>0,
$$

i.e., when the process of binding free growth factor particles to cells is superlinear.

For the kinetics given by (3.16) condition (3.11) is automatically satisfied in the model with $\mu=0$. For $\mu>0$ one might choose $\mu$ so large that (3.13) is not satisfied and therefore, there is no diffusion-driven instability of the spatially homogeneous steady state $(\bar{c}, \bar{b}, \bar{g})$.

To substantiate this claim in a special case, let us note the following result.

Lemma 3.4. Suppose that $\alpha(c)=\alpha c^{2}, 4 d_{c}>a_{1}$ and the following condition holds

$$
\mu>\frac{a_{1}^{2} \kappa}{d_{b}\left(4 d_{c}-a_{1}\right)} .
$$

Then no DDI occurs in the system (2.1)-(2.3).

Proof. The destabilisation condition with $\alpha(c)=\alpha c^{2}$ has the form $-a_{1} \bar{c}^{2} \bar{b}+\mu(\bar{c}+\bar{b})^{2}<0$. Since $\bar{c} \bar{b} \leq(\bar{c}+\bar{b})^{2} / 4$, a sufficient condition for absence of destabilisation is that $\mu>a_{1} \bar{c} / 4$. However, from Eq. (3.23) in Section 3.2., we obtain that

$$
-\frac{\mu}{a_{1} \bar{c}}+\frac{d_{c}}{a_{1}}=\frac{\kappa \bar{c}^{2}}{A(1+\bar{c})+\kappa \bar{c}^{2}+d_{b} \bar{c}^{2}(1+\bar{c})}<\frac{\kappa}{d_{b} \bar{c}},
$$

or equivalently that $\bar{c}<\left(\mu+a_{1} \kappa / d_{b}\right) / d_{c}$. Therefore, $\mu>a_{1} \bar{c} / 4$ is satisfied if $\mu>a_{1}(\mu+$ $\left.a_{1} \kappa / d_{b}\right) /\left(4 d_{c}\right)$, which is equivalent to $(3.17)$.

\subsection{Steady states of the kinetics system}

In the further analysis we assume the explicit form of $\alpha(c)=\alpha c^{2}$, which is the simplest function satisfying necessary condition (3.15). We have the steady states equations,

$$
\begin{aligned}
\left(\frac{a_{1} \bar{b}}{\bar{c}+\bar{b}}-d_{c}\right) \bar{c}+\mu & =0, \\
\alpha \bar{c}^{2} \bar{g}-d_{b} \bar{b}-d \bar{b} & =0, \\
-\alpha \bar{c}^{2} \bar{g}-d_{g} \bar{g}+d \bar{b}+\kappa(\bar{c}) & =b,
\end{aligned}
$$

From the second equation we obtain

$$
\bar{g}=\frac{d_{b}+d_{\bar{b}}}{\alpha \bar{c}^{2}}
$$


and by adding the second to the third equation and substituting (3.21)

$$
\bar{b}\left(\frac{d_{g}\left(d_{b}+d\right)}{\alpha \bar{c}^{2}}+d_{b}\right)=\kappa(\bar{c})
$$

and therefore

$$
\bar{b}=\frac{\kappa(\bar{c}) \bar{c}^{2}}{d_{b} \bar{c}^{2}+A},
$$

where $A=d_{g}\left(d_{b}+d\right) / \alpha$. Substituting this into the first equation, we obtain after some simplifications

$$
\frac{\kappa(\bar{c}) \bar{c}}{A+\kappa(\bar{c}) \bar{c}+d_{b} \bar{c}^{2}}=-\frac{\mu}{a_{1} \bar{c}}+\frac{d_{c}}{a_{1}} .
$$

For $\kappa$ being a Michaelis-Menten function of $c$, i.e., $\kappa(c)=\kappa c /(1+c)$, Eq. (3.22) is equivalent to

$$
f_{1}(\bar{c}) \equiv \frac{\kappa \bar{c}^{2}}{A(1+\bar{c})+\kappa \bar{c}^{2}+d_{b} \bar{c}^{2}(1+\bar{c})}=-\frac{\mu}{a_{1} \bar{c}}+\frac{d_{c}}{a_{1}} \equiv f_{2}(\bar{c}) .
$$

Continuity of $f_{1}$ and $f_{2}$, and $0=f_{1}(0)>f_{2}(0)=-\infty$ and $f_{1}(\infty)<f_{2}(\infty)$ imply that there exists at least one nonnegative root of Eq. (3.23). The number of nonnegative roots depends on the model parameters in the following way:

(1) if the maximum of the function $f_{1}$ is greater than $d_{c} / a_{1}$, then there exists a number $\mu^{*}$ such that for $\mu<\mu^{*}$ there exist three nonnegative roots of Eq. (3.23) (see Fig. 1), whereas for for $\mu>\mu^{*}$ there exists one nonnegative root of Eq. (3.23).

(2) if the maximum of the function $f_{1}$ is smaller than $d_{c} / a_{1}$, then Eq. (3.23) has also only one root for a very small $\mu$, and depending on the other parameters two scenarios are possible: (a) there exist such values of $\mu$ that for $\mu_{1}^{*}<\mu<\mu_{2}^{*}$ Eq. (3.23) has three roots (see Fig. 2), and (b) Eq. (3.23) has always only one root (see Fig. 3).

\subsection{Asymptotic bounds on solutions}

We first derive the equation for the total number of growth factor molecules. Adding equations $(2.2)-(2.3)$ side-by-side and integrating the resulting equation with respect to $x$, leads to

$$
\partial_{t} \int_{0}^{1}(b(t, x)+g(t, x)) d x=\int_{0}^{1} \kappa(c(t, x)) d x-\int_{0}^{1}\left(d_{b} b(t, x)+d_{g} g(t, x)\right) d x .
$$

Since $\kappa(c)$ is uniformly bounded by constant $\kappa$, we obtain that $\int_{\Omega}(b(t, x)+g(t, x)) d x$ is uniformly bounded from above.

Theorem 3.5.(i) Suppose that the condition

$$
Q=\frac{\kappa a_{1}}{d_{c} \min \left(d_{b}, d_{g}\right)}<1
$$



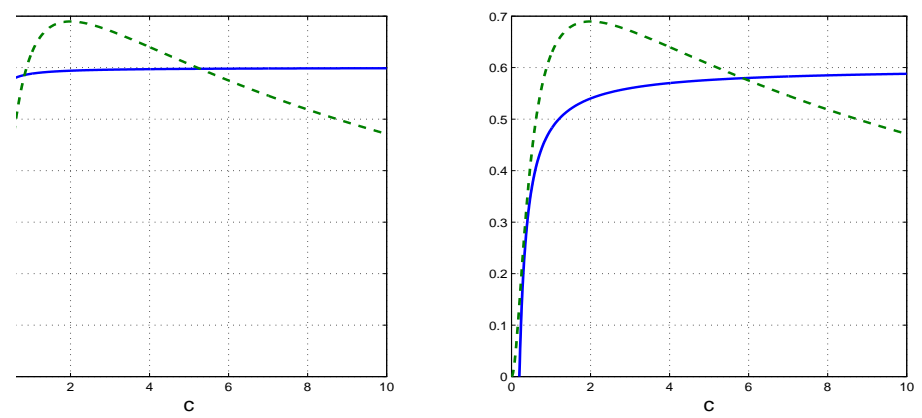

Figure 1: Plots of the functions $f_{1}(c)$ (dashed line) and $f_{2}(c)$ (solid line) in case, where the maximum of $f_{1}$ is greater than $d_{c} / a_{1}$ (for parameters: $a_{1}=1 / 12, d_{c}=0.05, d=d_{b}=d_{g}=$ $0.1, \kappa=1, \alpha=0.1$ ). We observe that there exist two regimes of the parameter value $\mu>0$. For $0<\mu<\mu^{*}$ the curves intersect in three points, and for $\mu>\mu^{*}$ they intersect in only one point. On the left-hand side we present graphs for $\mu=0.001$, and on the right-hand side for $\mu=0.01$.
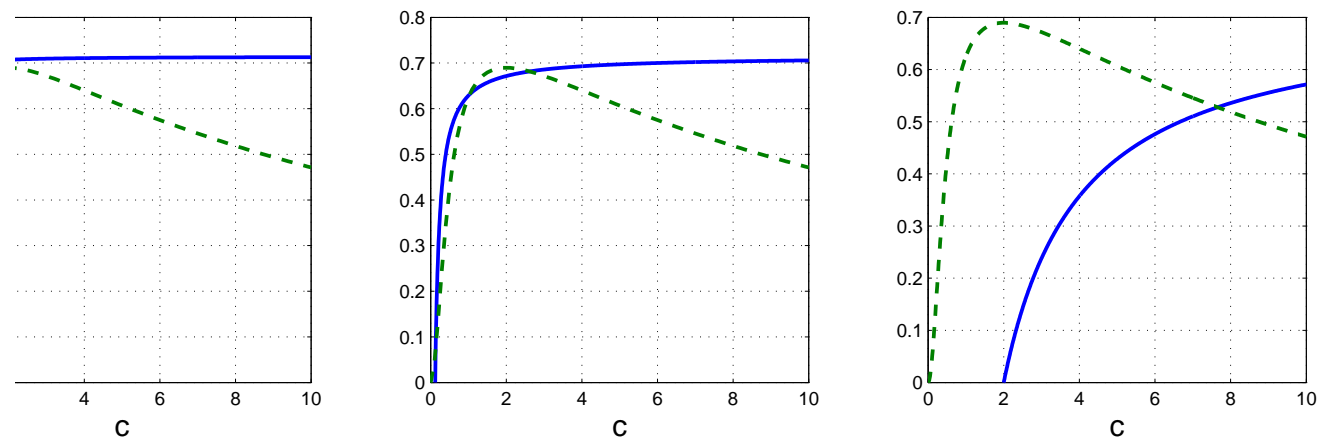

Figure 2: Plots of the functions $f_{1}(c)$ (dashed line) and $f_{2}(c)$ (solid line) in case, where the maximum of $f_{1}$ is smaller than $d_{c} / a_{1}$ (for parameters: $a_{1}=0.07, d_{c}=0.05, d=d_{b}=d_{g}=$ $0.1, \kappa=1, \alpha=0.1$ ). On the left-hand side we present graphs for $\mu=0.001$, in the center for $\mu=0.006$, and on the right-hand side for $\mu=0.1$. The pictures show three possible scenarios: for $0<\mu<\mu_{1}^{*} f_{1}$ and $f_{2}$ intersect in only one point, for $\mu_{1}^{*}<\mu<\mu_{2}^{*}$, the curves intersect in three points, and for $\mu>\mu_{2}^{*}$ they intersect in only one point. 

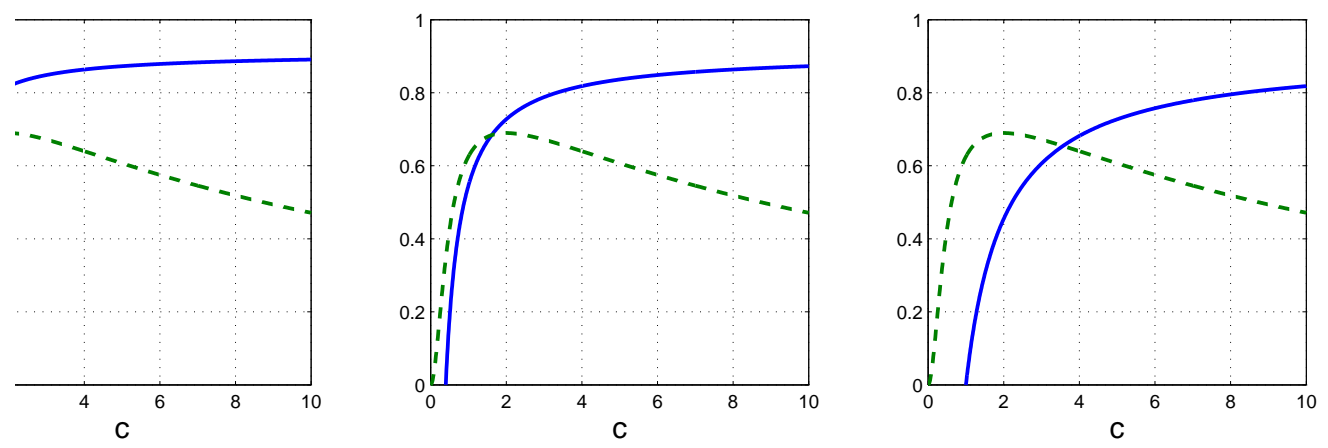

Figure 3: Plots of the functions $f_{1}(c)$ (dashed line) and $f_{2}(c)$ (solid line) in case, where the maximum of $f_{1}$ is smaller than $d_{c} / a_{1}$ (parameters: $a_{1}=0.055, d_{c}=0.05, d=d_{b}=d_{g}=0.1$, $\kappa=1, \alpha=0.1)$. On the left-hand side we present graphs for $\mu=0.01$, in the center for $\mu=0.02$, and on the right-hand side for $\mu=0.05$. The pictures show that independent on the value of $\mu, f_{1}$ and $f_{2}$ intersect in only one point.

is satisfied for the coefficients of the system (2.1)-(2.3) with $\kappa(c)=\kappa c /(1+c)$. Then,

$$
\underset{t}{\limsup } \int_{0}^{1} c(t, x) d x \leq \mu /\left[d_{c}(1-Q)\right]
$$

as $t \rightarrow \infty$. A corresponding upper bound on $\limsup _{t} B(t)$ can be deduced from the proof of the Theorem.

(ii) The following lower bounds hold,

$$
\begin{gathered}
\liminf _{t} c(t, x) \geq \mu / d_{c}, \\
\liminf _{t} \int_{0}^{1} c(t, x) d x \geq \mu / d_{c},
\end{gathered}
$$

and

$$
\liminf _{t} \int_{0}^{1}[b(t, x)+d(t, x)] d x \geq \kappa \mu /\left[\left(\mu+d_{c}\right) \max \left(d_{b}, d_{g}\right)\right] .
$$

Proof. (i) The nonlinear term of Eq. (2.1) can be estimated, uniformly in $c \geq 0$, by its asymptotic value of $b, \frac{c b}{c+b}<b(t, x)$. Therefore, we obtain the following inequality $\frac{\partial c(t, x)}{\partial t}+$ $d_{c} c(t, x) \leq a_{1} b(t, x)+\mu$ or, integrating with respect to $x$,

$$
\frac{d C(t)}{d t}+d_{c} C(t) \leq a_{1} B(t)+\mu
$$

where $C(t)=\int c(t, x) d x$ and $B(t)=\int b(t, x) d x$. Therefore, the solution satisfies

$$
C(t) \leq C\left(t_{0}\right) e^{-d_{c}\left(t-t_{0}\right)}+\int_{t_{0}}^{t} e^{-d_{c}(t-u)}\left[a_{1} B(u)+\mu\right] d u .
$$


Let us denote $\beta\left(t_{0}\right)=\sup _{u>t_{0}} B(u)$. The estimate above yields $C(t) \leq C\left(t_{0}\right) e^{-d_{c}\left(t-t_{0}\right)}+$ $\left[a_{1} \beta\left(t_{0}\right)+\mu\right] / d_{c}$. Taking lim $\sup _{t \rightarrow \infty}$ on both sides, we obtain $\lim \sup _{t} C(t) \leq\left[a_{1} \beta\left(t_{0}\right)+\mu\right] / d_{c}$. If this inequality holds for each $t_{0}$, then it also holds in the limit as $t_{0} \rightarrow \infty$ (which exists since $\beta\left(t_{0}\right)$ is nonnegative and nonincreasing). But $\beta(\infty)=\lim \sup _{t} B(t)$. Therefore, we eventually obtain

$$
\limsup _{t} C(t) \leq\left[a_{1} \limsup _{t} B(t)+\mu\right] / d_{c} .
$$

On the other hand, from (3.24) it follows that

$$
\frac{d[G(t)+B(t)]}{d t}+\min \left(d_{b}, d_{g}\right)[G(t)+B(t)] \leq \int \kappa c(t, x) /[1+c(t, x)] d x \leq \kappa C(t) .
$$

Using an analogous procedure, we obtain

$$
\limsup _{t} B(t) \leq \limsup _{t}[G(t)+B(t)] \leq\left[\kappa / \min \left(d_{b}, d_{g}\right)\right] \limsup _{t} C(t) .
$$

Combining (3.28) and (3.29), we obtain

$$
0 \leq \limsup _{t} C(t) \leq Q \limsup C(t)+\mu / d_{c}
$$

If the condition $Q<1$ is satisfied, then the above can be iterated to yield the assertion of part (i) the theorem.

(ii) Based on equation (2.1), we have

$$
\frac{\partial c}{\partial t}+d_{c} c \geq \mu
$$

which implies (3.25), based on reasoning as in the proof of Part (i). Integrating (3.30) sideby-side, with respect to $x$, we obtain $\partial \int_{0}^{1} c(t, x) d x / \partial t+d_{c} \int_{0}^{1} c(t, x) d x \geq \mu$, which yields (3.26). To obtain (3.27), we note that (3.24) implies

$$
\partial_{t}[B(t)+G(t)]+\max \left(d_{b}+d_{g}\right)[B(t)+G(t)] \geq \int_{0}^{1} \kappa \frac{c(t, x)}{1+c(t, x)} d x,
$$

which yields

$$
\begin{aligned}
\liminf _{t}[B(t)+G(t)] & \geq \liminf _{t} \int_{0}^{1} \kappa c(t, x) /[1+c(t, x)] d x \\
& \geq \int_{0}^{1} \kappa \liminf _{t} c(t, x) /[1+c(t, x)] d x=\kappa \mu /\left(\mu+d_{c}\right),
\end{aligned}
$$

where the second inequality follows by a version of Fatou Lemma, stating that for nonnegative $a(t, x), \liminf _{t \rightarrow \infty} \int_{0}^{1} a(t, x) d x \geq \int_{0}^{1} \liminf _{t \rightarrow \infty} a(t, x) d x$. 


\subsection{Existence of the inhomogeneous steady states}

We will use the same technique as in the previous paper [12], i.e., analysis of existence of periodic solutions of a second-order inhomogeneous steady-state ODE, satisfying the zeroflux boundary conditions. This equation is formally identical to that used by Murray in his works on pattern formation [19], however, here it emerges from a mixed-type system and therefore its explicit solution is difficult to find. We will find sufficient conditions for existence of periodic solutions and then verify they are satisfied in our system, under certain conditions on coefficients.

Theorem 3.6. A general solution of differential equation

$$
g^{\prime \prime}(x)=f[g(x)]
$$

with a $C^{\infty}$ function $f$, defined on an appropriate domain in $\mathbb{R}$, is given by a $C^{2}[0,1]$ function $g(x)$, implicitly defined by the relationship [6]:

$$
\int\left[C_{2}+2 \int f(g) d g\right]^{-1 / 2} d g=C_{1} \mp x .
$$

Existence of two branches of the solution reflects the fact the second derivative is invariant under sign change of $x$. Moreover, the sufficient condition for the solution of the "+" branch to satisfy the boundary conditions $g^{\prime}(0)=g^{\prime}(1)=0$ is that there exist $g^{* *}>g^{*}>0$ such that

$$
\int_{g^{*}}^{g} f(u) d u \geq 0, g \in\left(g^{*}, g^{* *}\right) ; \quad \int_{g^{*}}^{g^{* *}} f(u) d u=0
$$

and

$$
\int_{g^{*}}^{g^{* *}} F(u) d u=1
$$

where $F(u)=\left[2 \int_{g^{*}}^{u} f(v) d v\right]^{-1 / 2}$.

Proof. Calculate the first derivative of the solution defined by (3.32),

$$
\frac{d g}{d x}=\frac{1}{d x / d g}=\mp\left[C_{2}+2 \int f(g) d g\right]^{1 / 2}
$$

Repeated differentiation proves that, indeed, $g^{\prime \prime}=f(g)$. The boundary conditions are easily satisfied if the "+"-branch of solution is written in the form $x=\int_{g^{*}}^{g}\left[2 \int_{g^{*}}^{u} f(v) d v\right]^{-1 / 2} d u$.

Corollary 3.7. Consider equation (3.31) with the right-hand side defined as

$$
f_{a}(g)=a f(g), a>0 .
$$


If there exists an interval $\left(g_{+}, g_{-}\right)$such that $f_{a}\left(g^{+}\right)>0$ and $f_{a}\left(g^{-}\right)<0$, then there exists an $a>0$ such that the assertion of the Theorem holds.

Definition 3.8. (after [5], Definition 2.2) The local maximum $x_{0}$ of real function $f(x)$ of class $C^{2}$ is called a spike, if the nonlocal gradient of the first derivative of $f$, defined as

$$
\stackrel{\circ}{\nabla}_{\rho} f^{\prime}\left(x_{0}\right)=\frac{1}{2 \rho}\left[f^{\prime}\left(x_{0}+\rho\right)-f^{\prime}\left(x_{0}-\rho\right)\right]
$$

satisfies, for $\rho$ small enough, $\left|{ }^{\circ} \rho f^{\prime}\left(x_{0}\right)\right|>\left|f^{\prime \prime}\left(x_{0}\right)\right|$. The maximum is called a plateau if for $\rho$ small enough, it holds $\left|\stackrel{\circ}{\nabla}_{\rho} f^{\prime}\left(x_{0}\right)\right|<\left|f^{\prime \prime}\left(x_{0}\right)\right|$. The local minimum is called a spike (respectively, plateau) if $\left|\stackrel{\circ}{\nabla}_{\rho} f^{\prime}\left(x_{0}\right)\right|<\left|f^{\prime \prime}\left(x_{0}\right)\right|$ (respectively, $\left.\left|\stackrel{\circ}{\nabla}_{\rho} f^{\prime}\left(x_{0}\right)\right|>\left|f^{\prime \prime}\left(x_{0}\right)\right|\right)$.

The above criterion can be replaced by a simpler one, if the function $f$ is regular enough.

Corollary 3.9 (after [5], Theorem 2.3) Assume $f \in C^{5}$, and $x_{0}$ is an extremum with $f^{\prime \prime}\left(x_{0}\right) \neq 0 f^{i v}\left(x_{0}\right) \neq 0$. Then, the extremum is a spike (respectively, a plateau) if $f^{i v}\left(x_{0}\right)$ has a different (respectively, the same) sign as $f^{\prime \prime}\left(x_{0}\right)$.

The corollary above allows to relate the intuitive notion of a "spiky" (sharp) or "plateau-like" extremum to verifiable mathematical conditions. This is one of the ways in which numerical simulations can be mathematically verified (compare Figs. 4 and 8).

Based on Theorem 3.6 and its corollary, it is possible to prove the following version of the existence result for the periodic inhomogeneous equilibria. The proof is almost identical to that in our previous paper [13]. The Theorem is used to guarantee the existence of periodic spatially-inhomogeneous equilibria, such as the one depicted in Figure 4.

Theorem 3.10. Suppose that $g(x)$ is the increasing branch of the solution to Eq. (3.31), on the interval $x \in[0,1]$, satisfying conditions $g^{\prime}(0)=g^{\prime}(1)=0$ and $g(0)=g^{*}<g(1)=g^{* *}$. Let us choose natural number $n$, such that $n \geq 1$. Then, the function $h(x)$, defined on $x \in[0,1]$, and such that

$$
\left.h\right|_{[(k-1) /(2 n), k /(2 n)]}=h_{k}, \quad k=1,2, \ldots, 2 n,
$$

where

$$
\begin{aligned}
& h_{k}(u)=g(k-2 n u), \quad u \in[(k-1) /(2 n), k /(2 n)], \quad k \text { odd }, \\
& h_{k}(u)=g(2 n u-k+1), \quad u \in[(k-1) /(2 n), k /(2 n)], \quad k \text { even, }
\end{aligned}
$$

is of class $C^{5}[0,1]$ and it satisfies the following equation

$$
\frac{1}{4 n^{2}} h^{\prime \prime}(u)=f[h(u)]
$$

on $u \in[0,1]$. 
Moreover,

$$
\begin{array}{ll}
h\left(\frac{k}{2 n}\right)=g^{*}, \quad h^{\prime}\left(\frac{k}{2 n}\right)=0, \quad h^{\prime \prime}\left(\frac{k}{2 n}\right)>0, \quad h^{\prime \prime \prime}\left(\frac{k}{2 n}\right)=0, \quad h^{i v}\left(\frac{k}{2 n}\right)=f^{\prime}\left[h\left(\frac{k}{2 n}\right)\right] h^{\prime \prime}\left(\frac{k}{2 n}\right), \quad \text { k odd }, \\
h\left(\frac{k}{2 n}\right)=g^{* *}, \quad h^{\prime}\left(\frac{k}{2 n}\right)=0, \quad h^{\prime \prime}\left(\frac{k}{2 n}\right)<0, \quad h^{\prime \prime \prime}\left(\frac{k}{2 n}\right)=0, \quad h^{i v}\left(\frac{k}{2 n}\right)=f^{\prime}\left[h\left(\frac{k}{2 n}\right)\right] h^{\prime \prime}\left(\frac{k}{2 n}\right), \quad \text { k even },
\end{array}
$$

This Theorem implies that if $f^{\prime}\left[h\left(\frac{k}{2 n}\right)\right]$ is positive (respectively, negative), then the solution $h$ has an extremum at $u=k /(2 n)$, which is a plateau (respectively, a spike).

Remark 3.11. Let us note that the numerical periodic spatially inhomogeneous solution $g(x)$ depicted in Figure 4 has maxima, which are plateaux and minima, which are spikes. This is confirmed by a numerical study of the derivatives of function $f(g)$ at the extrema.
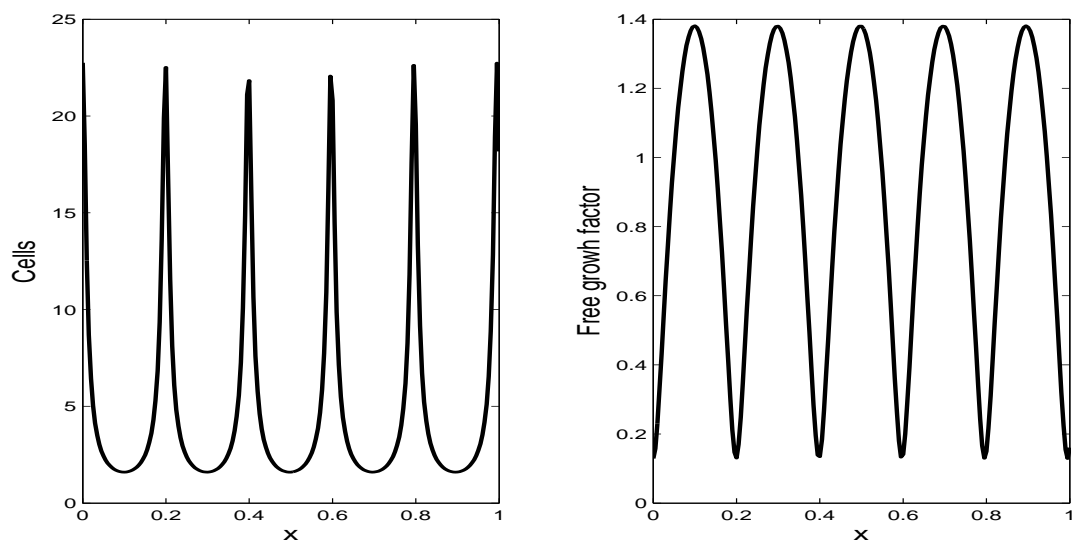

Figure 4: Spatial profile of the periodic equilibrium spatially inhomogeneous solutions $c$ and $g$ of model (2.1)-(2.3) with parameters as in Fig. 7, except for $\gamma=1000$. The graph was obtained as a solution of the equilibrium equation (3.43).

We will now apply the results stated above to demonstrate that there exist coefficient values in the equations (2.1)-(2.3) such that inhomogeneous steady states exist. We will reduce the problem to existence of solutions of a second-order ODE such as in Theorem 3.10. Spatially inhomogeneous steady states of the system (2.1)-(2.3) satisfy the following system of equations

$$
\begin{aligned}
0 & =\left(\frac{a_{1}}{1+c / b}-d_{c}\right) c+\mu, \\
0 & =\alpha c^{2} g-d_{b} b-d b, \\
0 & =\frac{1}{\gamma} g^{\prime \prime}(x)-\alpha c^{2} g-d_{g} g+\kappa \frac{c}{c+1}+d b,
\end{aligned}
$$

where now $c=c(x), b=b(x)$, and $g=g(x)$. Adding equations (3.41) and (3.42) side-by-side, we obtain

$$
\frac{1}{\gamma} g^{\prime \prime}(x)=d_{g} g+d_{b} b-\kappa \frac{c}{c+1} .
$$


From equation (3.40), we obtain $b=c\left(c d_{c}-\mu\right) /\left[c\left(a_{1}-d_{c}\right)+\mu\right]$, which after substitution into (3.41) yields the following quadratic equation for $c$ as a function of $g$

$$
\alpha g\left(a_{1}-d_{c}\right) c^{2}+\left[\alpha \mu g-\left(d_{b}+d\right) d_{c}\right] c+\left(d_{b}+d\right) \mu \equiv A c^{2}+B c+C=0,
$$

which has real roots if the discriminant $\Delta_{c}$ is nonnegative

$$
\Delta_{c}=\left[\alpha \mu g-\left(d_{b}+d\right) d_{c}\right]^{2}-4 \alpha \mu g\left(a_{1}-d_{c}\right)\left(d_{b}+d\right) \geq 0 .
$$

This latter is a quadratic polynomial in $g$, with a discriminant $\Delta_{g}=4 \alpha^{2} \mu^{2}\left(d_{b}+d\right)^{2}\left(4 a_{1}^{2}-\right.$ $4 a_{1} d_{c}$ ), which is positive if only $a_{1}>d_{c}$. Therefore, $\Delta_{c}$ is nonnegative if either $g<g_{1}$ or $g>g_{2}$, where $g_{1,2}=2 \frac{\left(d_{b}+d\right) a_{1}}{\alpha \mu}\left[\left(1-d_{c} / 2 a_{1}\right) \mp \sqrt{1-d_{c} / a_{1}}\right]>0$. If $g>g_{2}$, then under $a_{1}>d_{c}$, also $g>\left(d_{b}+d\right) d_{c} /(\alpha \mu)$, which means that $B>0$. However the roots of (3.44) have the form

$$
c_{1,2}=\frac{-B \mp \sqrt{B^{2}-4 A C}}{2 A}
$$

which means that in view of the fact that under $a_{1}>d_{c}$, it holds $A, C>0$, and therefore $c_{1,2}>0$ iff $B<0$. Therefore, the only possibility remains that $g<g_{1}$. We verify that $c_{1}>0$ if $g \in\left(0, g_{1}\right)$. This requires $B<0$, i.e., $g<g_{0}$, where $g_{0}=\left(d_{b}+d\right) d_{c} /(\alpha \mu)$. If we consider the ratio $g_{1} / g_{0}=2\left(a_{1} / d_{c}\right)\left[1-d_{c} /\left(2 a_{1}\right)-\sqrt{1-d_{c} / a_{1}}\right]$, we see that it is of the order of $2 o\left(d_{c} / a_{1}\right) /\left(d_{c} / a_{1}\right)$, which is less than 1 if only $d_{c} / a_{1}$ is small enough.

Let us note the following identities:

$$
\begin{gathered}
c_{1,2}^{2}=-\frac{B c_{1,2}+C}{A}, \\
c_{1,2} /\left(1+c_{1,2}\right)=\frac{C+A c_{1,2}}{A-B+C} .
\end{gathered}
$$

Substituting the above into (3.43), taking into account that $b=\alpha c^{2} g /\left(d_{b}+d\right)$, and choosing branch $c_{2}$, we obtain

$$
\frac{1}{\gamma} g^{\prime \prime}(x)=d_{g} g-\frac{\alpha d_{b} g}{d_{b}+d} \frac{B c_{2}+C}{A}-\kappa \frac{C+A c_{2}}{A-B+C} \equiv f(g)
$$

Constant $\gamma$ plays the same role as constant $a$ in Corollary 3.7, therefore it is enough to prove that there exists an subinterval $\left(g_{+}, g_{-}\right)$of the domain on which $f(g)$ is well-defined, such that $f\left(g^{+}\right)>0$ and $f\left(g^{-}\right)<0$. Let us notice that $f(g)$ has a pole at $A=0$ or equivalently at $g=0$. Also $f(0+)=\infty$, which means that we can find a suitable $g^{+}$. On the other hand, there exists a pole at $A-B+C=0$, however, this latter occurs for a negative $g$ if $\mu$ is small enough, eg. if $\mu<a_{1}-d_{c}$. Also, we can select $g^{-}=g_{1}$, since if $g=g_{1}$ then $B=-2 \sqrt{A C}$ and $c_{2}\left(g_{1}\right)=-B /(2 A)=\sqrt{C / A}$ so that we obtain that

$$
f\left(g_{1}\right)=d_{g} g_{1}+\frac{\alpha d_{b} g_{1}}{d_{b}+d} \frac{C}{A}-\kappa \frac{2 C-B}{2(A-B+C)} .
$$


The term $\frac{2 C-B}{2(A-B+C)}$ has numerator and denominator positive if $\mu$ is small enough, therefore, $f\left(g_{1}\right)<0$ if $\kappa$ is large enough. In summary, we have the following corollary.

Corollary 3.12. For any $n \geq 1$ there exists a value of diffusion constants $1 / \gamma$, such that inhomogeneous periodic steady states with period $1 / n$ exist in the system (2.1)-(2.3), if $\mu$ and $d_{c}$ are small enough and $\kappa$ is large enough. Estimates for $\mu, d_{c}$ and $\kappa$ can be provided, based on the detailed derivations, which are notationally complicated.

Numerical studies (not shown) indicate that the sufficient conditions provided in the Corollary 3.12, are by far not necessary. However, the number of special cases to consider seems to make a more accurate analytical result impractical.

\section{Numerical computations}

We simulate solutions of the model with parameters coresponding to different scenarios of the number and stability of the spatially homogeneous steady states, as depicted in Figs. 1-3. Numerical simulations illustrate the role of cell influx represented by the parameter $\mu>0$. In the qualitative behaviour of the model, the magnitude of $\mu$ plays a major role, in that it determines the number of equilibria of the kinetics system. As explained in Section 3.2., two special cases can be distinguished, depending on whether the ratio $d_{c} / a_{1}$ is greater or smaller compared to the maximum of function $f_{1}$, defined in expression (3.23).

If the maximum of function $f_{1}$ is greater than $d_{c} / a_{1}$, then there exist three nonnegative roots for $\mu$ below a threshold (see Fig. 1 left panel) and one nonnegative root for $\mu$ above this threshold (see Fig. 1, right panel). The exact pattern of stability of these equilibria seems to be difficult to investigate analytically because of the complicated form of algebraic conditions for stability. Numerically, it appears that the smallest nonnegative steady state is always stable, the second one is always unstable, and the third steady state (the greatest one) satisfies DDI conditions (3.8)-(3.11) and can be destabilised by diffusion. Numerical simulations indicate transient growth of of spike-like solutions, which finally decay and converge to a unique stable spatially homogeneous equilibrium (see Fig. 5).

When $\mu$ is larger than the threshold only one spatially homogeneous equilibrium exists. The stability of this state depends on the value of $\mu$ (Corollary 3.12). There exists another threshold such that for large enough $\mu$, destabilisation condition (3.11) is not satisfied (c.f. also Lemma 3.4). Accordingly, no destabilisation of the constant solution is observed (see Fig. 6).

For $\mu$ between the two thresholds DDI conditions (3.8)-(3.11) are fulfiled and the destabilisation of the spatially homogeneous state leads to the formation of the stationary spike-like patterns (see Figs. 7-8). The number of "spikes" depends on the scaling coefficient $\gamma$ and, in some cases, on the initial perturbation in similar manner as in our previous models. These issues were discussed in [13]. 

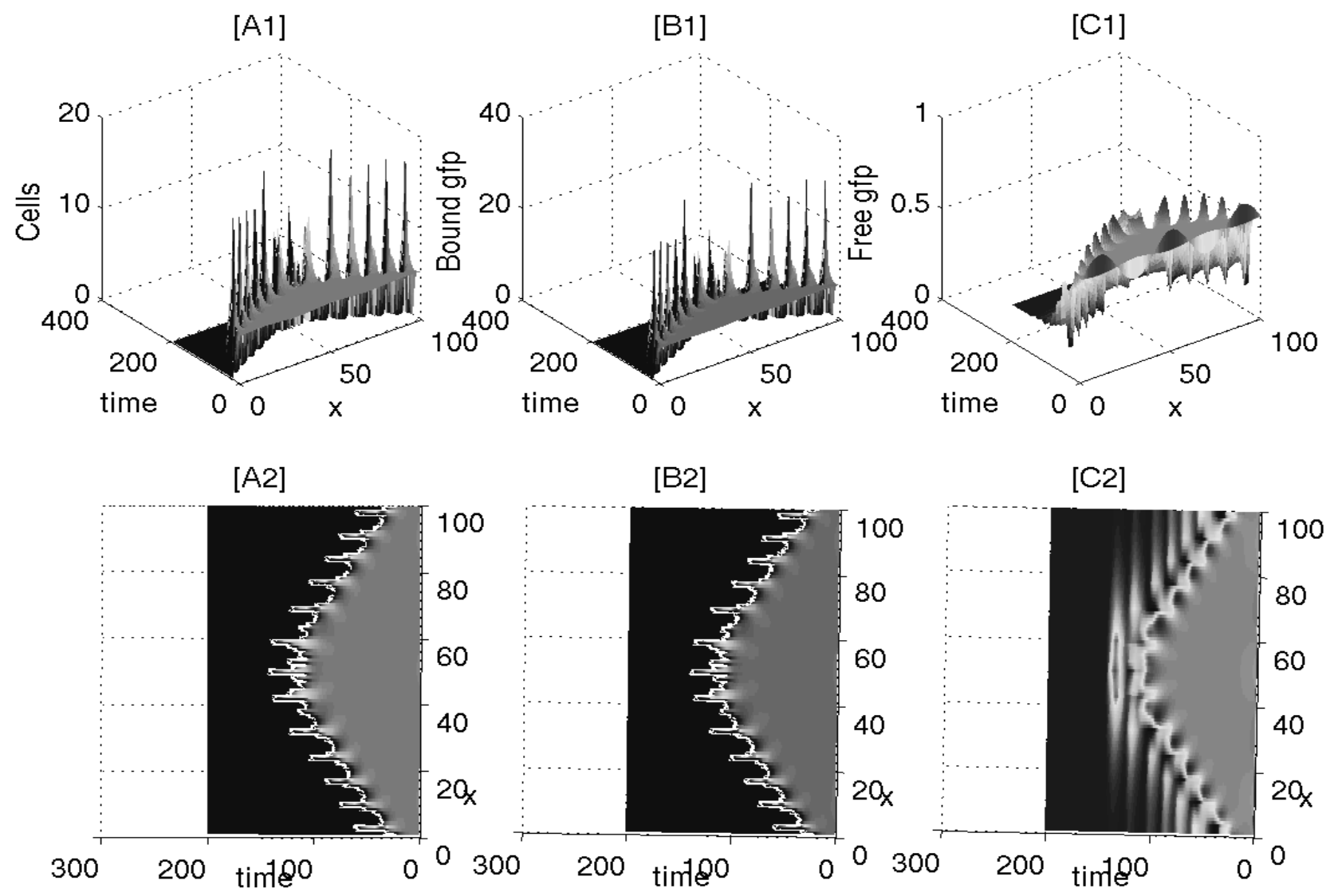

[B2]

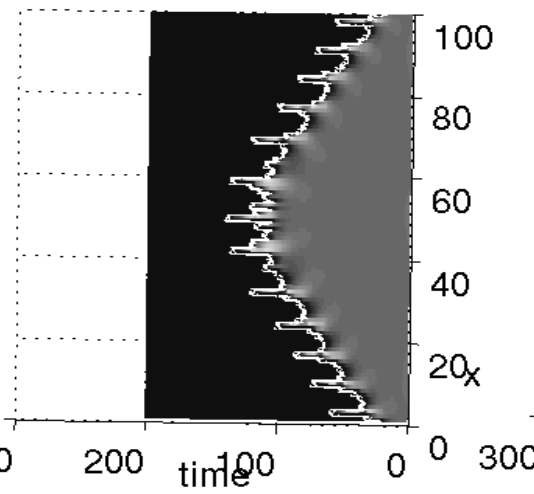

[C2]

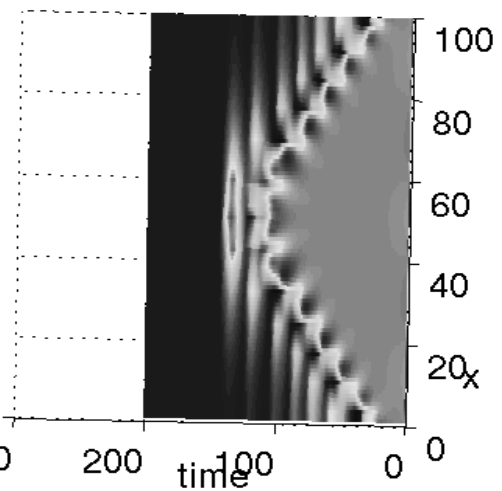

Figure 5: Time evolution of solutions of model (2.1)-(2.3) with parameters: $a_{1}=1 / 12$, $d_{c}=0.05, d=d_{b}=d_{g}=0.1, \kappa=1, \alpha=0.1$ and $\mu=0.001$ and scaling coefficient $\gamma=100: c(x, t)$ left, $b(x, t)$ center, $g(x, t)$ right. The upper and the lower panel show the same solutions but from the different perspective. The parameterers correspond to the case, when there exist three nonnegative spatially homogeneous steady states (as in Fig. 1 left panel). We observe a destabilisation of the spatially homogeneous steady state, but the solutions converge to the smallest spatially homogeneous state. In biological terms, this means the emerging cancerous cell populations are not persistent. 

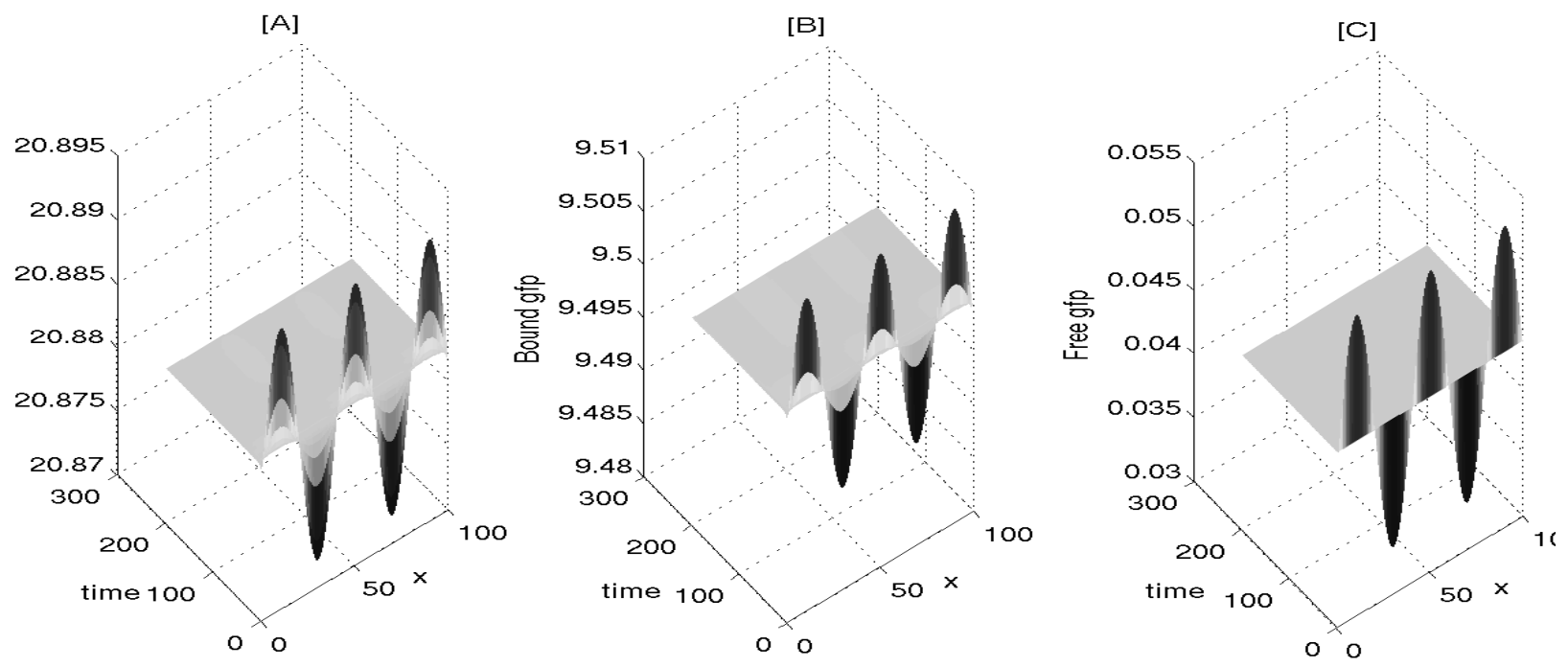

Figure 6: Time evolution of solutions of model (2.1)-(2.3): $c(x, t)$ left, $b(x, t)$ center, $g(x, t)$ right. Parameters are identical as in Fig. 5 but with greater $\mu=0.5$, such that there exists only one nonnegative spatially homogeneous steady states (similar as in Fig. 1 right panel) but the DDI condition is not fulfiled. We observe that there is no destabilisation of the spatially homogeneous steady state.

Let us notice, that as opposed to the model in the previous paper [12], where no influx of mutated cells was assumed $(\mu=0)$, apparently stable spatially inhomogeneous limit states appear in the case of Michaelis-Menten-type function $\kappa(c)$ and no additional assumption on the constant influx of growth factor particles is needed.

If the maximum of the function $f_{1}$ is smaller than $d_{c} / a_{1}$, then the situation is more complicated. Whereas there is always at least one nonnegative root, there may exist an interval of $\mu$, such that for $\mu$ inside this interval, three roots exist. The variants possible are depicted in Figs. 2 and 3. For the case of $d_{c} / a_{1}$ large compared to the maximum of the function $f_{1}$, when $\mu$ is small, there exists always only one spatially homogeneous solution, which is always stable (Fig. 2 left panel). If there exists an interval of $\mu$, for which three spatially homogeneous equilibria exist (Fig. 2 center panel), the largest equilibrium is unstable to the spatially heterogeneous perturbations. Interestingly, the long time dynamics in this case (persistence of spatial pattern or decay to the spatially constant equilibrium) also may depend on the scaling coefficient $\gamma$ (see Fig. 9).

If $\mu$ is larger and such that again only one spatially homogeneous equilibrium exists (Fig. 2 right panel), solutions of the model converge to the stationary spike-like pattern (see Fig. 10).

Another interesting case is the one in which, independently on the values of $\mu$, there always exists only one nonnegative spatially homogeneous steady state (as depicted in Fig. 3). Such steady state is always stable. 

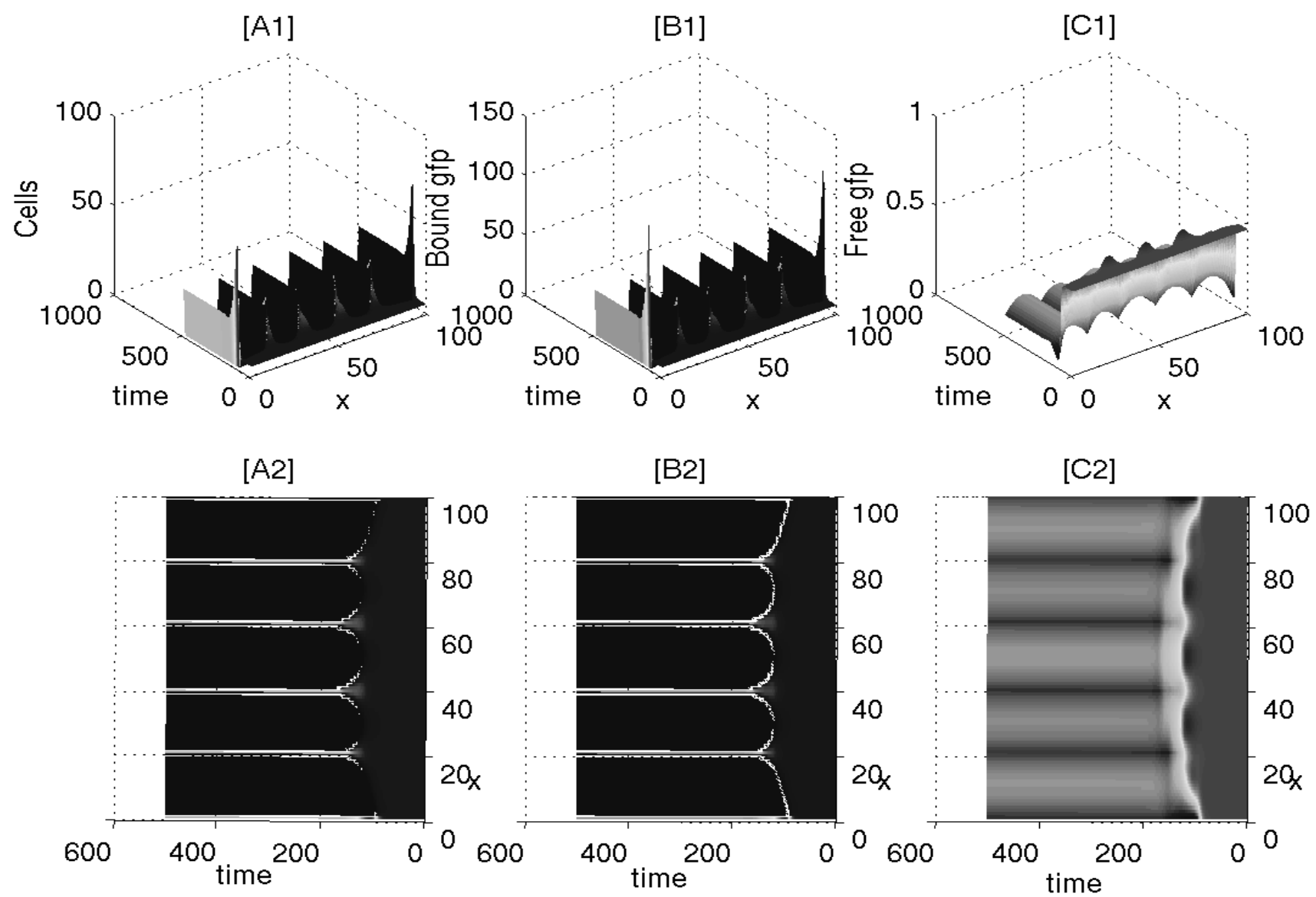

Figure 7: Time evolution of solutions of model (2.1)-(2.3): $c(x, t)$ left, $b(x, t)$ center, $g(x, t)$ right. The upper and the lower panel show the same solutions but from the different perspective. Parameters are identical as in Fig. 5 but with greater $\mu=0.01$, such that there exists only one nonnegative spatially homogeneous steady state (as in Fig. 1 right panel and the steady state fulfils the DDI condition. We observe a destabilisation of the spatially homogeneous steady state that leads to the growth of stable spike-like solutions. 

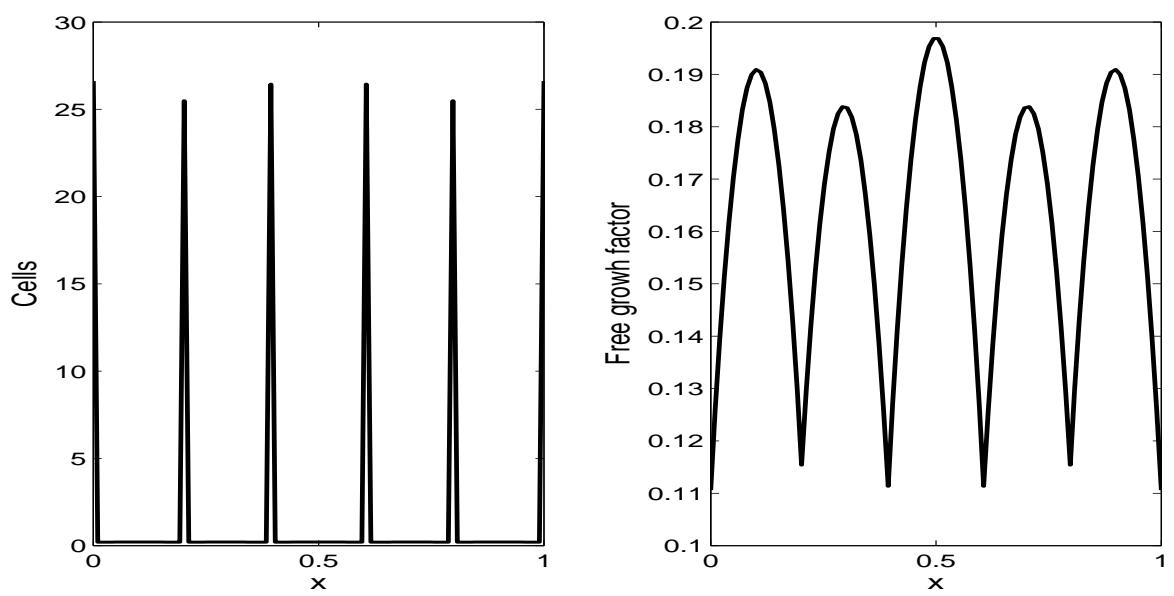

Figure 8: Spatial profile of the limit spatially inhomogeneous solutions $c$ and $g$ of model (2.1)-(2.3) with parameters as in Fig. 7. The graph was obtained from long-time simulation of the model.
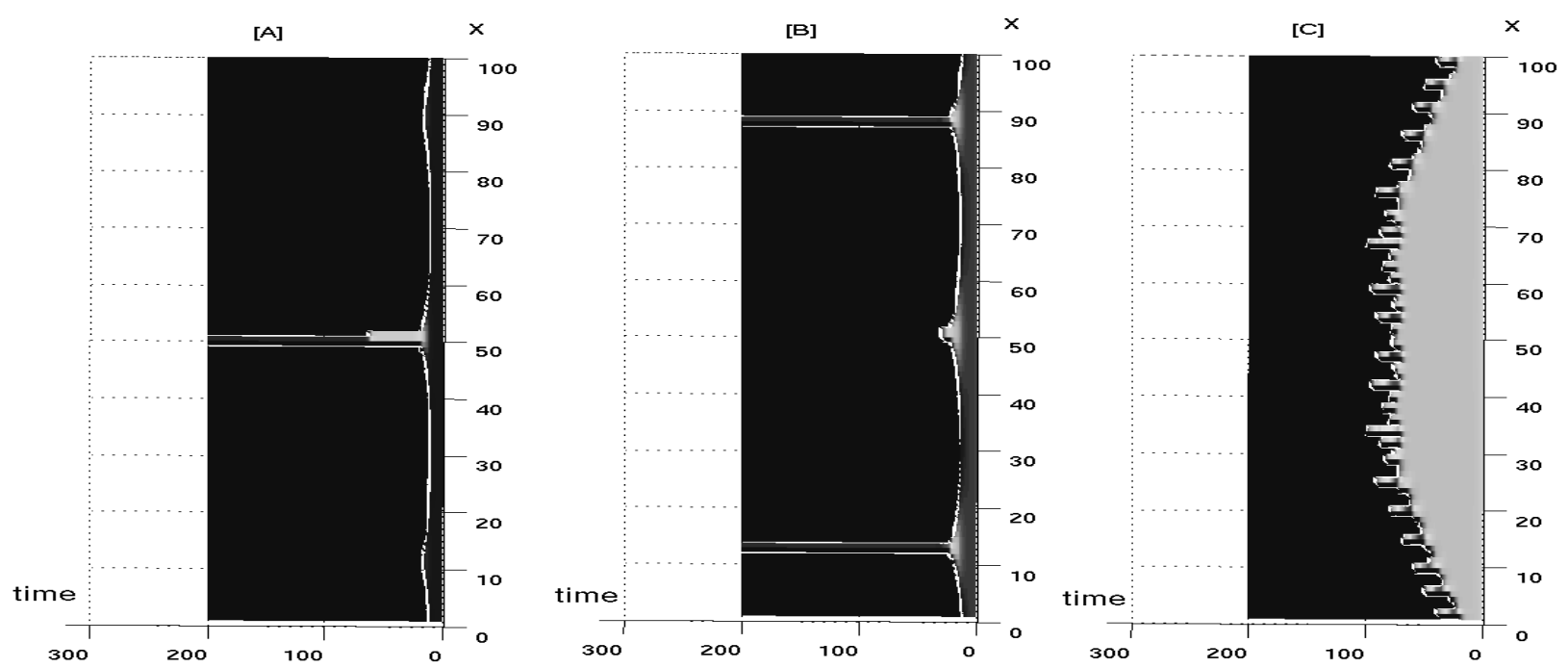

Figure 9: Time evolution of cell density $c(x, t)$, solution of model (2.1)-(2.3) with parameters: $a_{1}=0,07, d_{c}=0.05, d=d_{b}=d_{g}=0.1, \kappa=1, \alpha=0.1$ and $\mu=0.006$ and different scaling coefficients: $\gamma=1$ left, $\gamma=100$ center, $\gamma=10000$ right. Parameters correspond to the case presented in in Fig. 2, center panel. We observe a destabilisation of the spatially homogeneous steady state (the largest one) and growth of spike-like solutions, which are not stable and decay to the spatially homogeneous steady state for large scaling coefficient (small diffusion) and seem to be stable for a smaller scaling coefficient. 

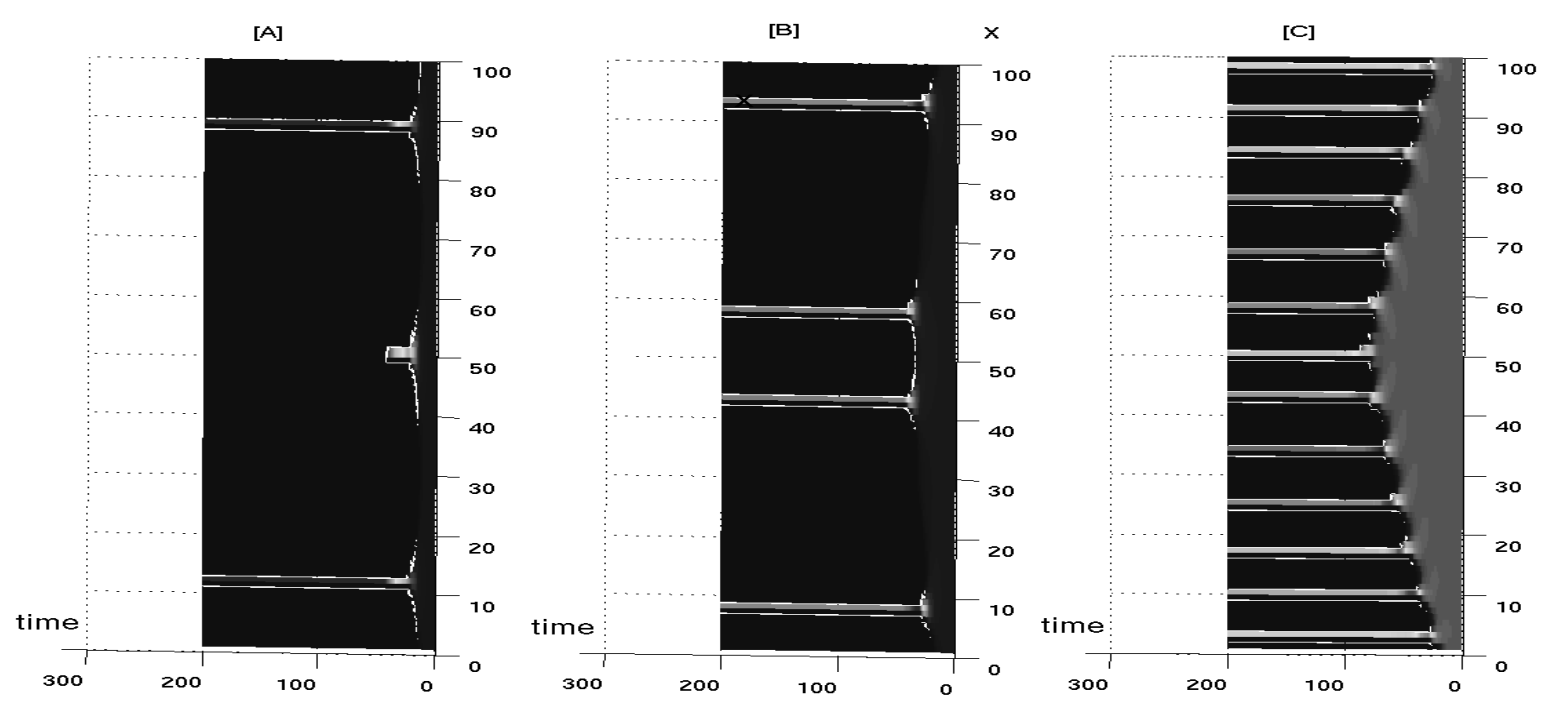

Figure 10: Time evolution of cell density $c(x, t)$, solution of model $((2.1)-(2.3)$ with parameters identical as in Fig. 9, except for $\mu=0.1$. Parameters correspond to the case presented in in Fig. 2, right panel. Scaling coefficients are : $\gamma=10$ left, $\gamma=100$ center, $\gamma=1000$ right. We observe a destabilisation of the spatially homogeneous steady state and growth of spike-like solutions, which persist in time independently on the scaling coefficient.

\section{Discussion}

We consider a model, in which pre-malignant cells are supplied via mutation from the normal cell population. For simplicity, the mutation rate is assumed constant and uniformly distributed over the spatial domain (unit interval). Previously, in references [12, 13, 14], we considered models, in which no cell influx was present. These earlier models all had a trivial equilibrium, which attracted solutions unless external supply of growth factors was assumed. As a result, even when the diffusion destabilisation was present, the emerging spatially distributed colonies were frequently transient. Biologically, these models corresponded to the situation in which a single colony of pre-malignant cells, once established, may stabilise or die out.

The current model lacks the trivial equilibrium. It can display, as explained in Section 4. from one to three nontrivial equilibria. The solutions represent a variety of behaviours, also discussed in Section 4. The prominent role of parameter $\mu$ emerges from this analysis. This is confirmed by the Theorem 3.5, which provides asymptotic bounds on solutions (in the limes superior and limes inferior sense). Part (i) of this results shows that as parameter $\mu$ becomes small, the upper bound (in the sense of limes superior of the $L^{1}$-norm) on solutions also becomes small and equal to 0 when $\mu=0$ (as was the case in models in $[12,13,14]$ ). Interestingly, the pointwise lower bound on limes inferior becomes close to the $L_{1}$ upper bound on limes superior, when the factor $Q$ approaches zero. In such situation, either the amplitude of oscillations becomes small, or the spatially inhomogeneous steady state includes 
very narrow spike-type peaks separated by wide and shallow troughs. When $Q$ becomes equal to zero, for example when $\kappa=0$, both bounds are identical and equal to $\mu / d_{c}$. This means the solution converges to this value, the fact which is intuitively obvious, but provides a consistency check on Lemma 3.4 (condition of for no DDI is automatically satisfied when $\kappa=0)$ and Corollary $3.12\left(f\left(g_{1}\right)>0\right.$ when $\left.\kappa=0\right)$.

Numerical simulations suggest that the diffusion-driven instabilities emerging in the model may be stable (Section 4.). whether or not this happens, depends on the number and stability of spatially homogeneous steady states. If there exists only one spatially homogeneous state and the state satisfies conditions for DDI, then the solutions tend to the stationary spike-like pattern. This is corroborated by existence of spatially inhomogeneous steady states, as discussed in Section 3.4. (see Fig. 4). As stated in Theorem 3.10, the inhomogeneous steady states have extrema of spike or plateau type, depending on the sign of the derivative of $f$ at the extremum. Due to a complicated form of this function, this condition can be only numerically verified. An example is discussed in Remark 3.11.

Another novelty in the dynamics of the model solution is that small changes of parameter $a_{1}$ may change qualitative behaviour of the model solutions. As shown in numerical simulations even for the same values of $\mu$ small change of $a_{1}$ may change the stability of the spatially homogeneous steady states, and for example a stable state can become unstable for the spatially heterogeneous perturbations leading to the emergence of the stable spatial patterns. It corresponds to the situation, when a small increase of the proliferation rate leads to the destabilisation of cell population, even if the influx of mutated cells is unchanged.

Biologically, we obtain behaviour, which seems to be consistent with the field theory of carcinogenesis. Lesions started by pre-malignant cells are frequently transient (as in the model in the previous work [13]). However, when there is a constant low-level supply of these founder cells, due to mutation of normal cells (the current model), a stable distribution is obtained, which is spatially inhomogeneous and sensitive to initial conditions. When the mutation rate is large, which is an unrealistic scenario (typical mutation rates in mammalian cells are below $10^{-6}$ per division, c.f. [9] and references therein), the system saturates and produces only asymptotically spatially homogeneous solutions. The current model is an intermediate step between the model of references $[12,13,14]$, in which just a single premalignant colony was considered, and a comprehensive model, which should involve stabilisation of some of the pre-malignant colonies by means of progressive deregulation of the genome. This model will be subject of further studies.

It seems appropriate to stress once more the critical role of diffusion of free growth factors in emergence of spatial patterns similar to those observed in early carcinogenesis. As discussed in [13], models taking diffusion into account may behave in a way, which is intuitively difficult to predict and leads to different conclusions.

\section{Acknowledgements}

Marek Kimmel was supported by the NCI/CISNET grant U01CA097431B. Anna MarciniakCzochra was supported by Center for Modeling and Simulation in the Biosciences (BIOMS) 
in Heidelberg and WIN-Kolleg of the Heidelberg Academy of Science and Humanities.

\section{References}

[1] H. Awaya, Y. Takeshima, O. Furonaka, N. Kohno, K. Inai. Gene amplification and protein expression of EGFR and HER2 by chromogenic in situ hybridisation and immunohistochemistry in atypical adenomatous hyperplasia and adenocarcinoma of the lung. J. Clin. Pathol., 58 (2005), 1076-1080.

[2] R. Axelrod, D. E. Axelrod, K. J. Pienta. Evolution of cooperation among tumour cells. Proc. Nat. Acad. Sci., 103 (2006), 13474-13479.

[3] K. Chueh, C. Conley, J. Smoller. Positively invariant regions for systems of nonlinear diffusion equations. Ind. Univ. Math. J., 26 (1977), 373-392.

[4] D. Henry. Geometric theory of semilinear parabolic equations. Springer-Verlag, 1981.

[5] T. Hillen. A classification of Spikes and Plateaus. SIAM Review, 2007, in press.

[6] E. Kamke. Differentialgleichungen: Losungsmethoden und Losungen. In: Gewohnliche Differentialgleichungen B. G. Teubner, 1977.

[7] T. Kawai, S. Hiroi, K. Nakanishi, A. K. Meeker. Telomere length and telomerase expression in atypical adenomatous hyperplasia and small bronchioloalveolar carcinoma of the lung. Am. J. Clin. Pathol., 127 (2007), 254-262.

[8] K. M. Kerr. Pulmonary preinvasive neoplasia. J. Clin. Pathol., 54 (2001), 257-271.

[9] M. Kimmel, D. E. Axelrod. Fluctuation test for two-stage mutations: application to gene amplification. Mutat. Research, 306 (1994), 45-60.

[10] K. Kishi, S. Homma, A. Kurosaki, S. Tanaka, H. Matsushita, K. Nakata. Multiple atypical adenomatous hyperplasia with synchronous multiple primary bronchioloalveolar carcinomas. Intern. Med., 41 (2002), 474-477.

[11] H. Kitagawa, A. Goto, T. Niki, M. Hironaka, J. Nakajima, M. Fukayama. Lung adenocarcinoma associated with atypical adenomatous hyperplasia. A clinicopathological study with special reference to smoking and cancer multiplicity. Pathol. Int., 53 (2003), 823827.

[12] A. Marciniak-Czochra, M. Kimmel. Modelling of early lung cancer progression: Influence of growth factor production and cooperation between partially transformed cells. Math. Mod. Meth. Appl. Sci., 17 (2007), 1693-1719.

[13] A. Marciniak-Czochra, M. Kimmel. Dynamics of growth and signalling along linear and surface structures in very early tumours. Comp. Math. Meth. Med., 7 (2006), 189-213. 
[14] A. Marciniak-Czochra, M. Kimmel. Reaction-diffusion approach to modelling of the spread of early tumours along linear or tubular structures. J. Theor. Biol., 244 (2006), 375-387.

[15] A. Marciniak-Czochra, M. Kimmel. Mathematical model of tumour invasion along linear or tubular structures. Math. Comp. Modelling, 41 (2005), 1097-1108.

[16] A. Marciniak-Czochra, M. Ptashnyk. Derivation of a macroscopic receptor- based model using homogenisation techniques. SIAM J. Math. Anal., 2008, to appear.

[17] A. Marciniak-Czochra. Developmental models with cell surface receptor densities defining morphological position. PhD thesis, University of Heidelberg, 2004.

[18] L. Morandi, S. Asioli, A. Cavazza, A. Pession, S. Damiani. Genetic relationship among atypical adenomatous hyperplasia, bronchioloalveolar carcinoma and adenocarcinoma of the lung. Lung Cancer, 56 (2007), 35-42.

[19] J. D. Murray. Mathematical biology. Springer-Verlag, Berlin, 2003.

[20] F. Rothe. Global solutions of reaction-diffusion systems. Springer-Verlag, Berlin, 1984.

[21] T. Sano, Y. Kitayama, H. Igarashi, M. Suzuki, F. Tanioka, K. Chida, K. Okudela, H. Sugimura. Chromosomal numerical abnormalities in early stage lung adenocarcinoma. Pathol. Int., 56 (2006), 117-125.

[22] J. Smoller. Shock-waves and reaction-diffusion equations. Springer-Verlag, Berlin, 1994.

[23] K. Takamochi, T. Ogura, K. Suzuki, H. Kawasaki, Y. Kurashima, T. Yokose, A. Ochiai, K. Nagai, Y. Nishiwaki, H. Esumi. Loss of heterozygosity on chromosomes $9 q$ and $16 p$ in atypical adenomatous hyperplasia concomitant with adenocarcinoma of the lung. Am. J. Pathol., 159 (2001), 1941-1948. 